\title{
A-Z of malignant peripheral nerve sheath tumors
}

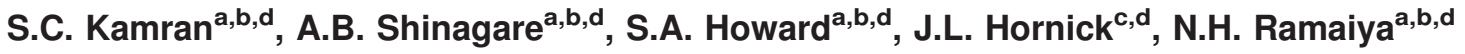 \\ ${ }^{a}$ Department of Imaging, Dana-Farber Cancer Institute, 450 Brookline Ave, Boston, MA 02115, USA; \\ ${ }^{b}$ Department of Radiology, Brigham and Women's Hospital, 75 Francis Street, Boston, MA 02115, USA; \\ ${ }^{c}$ Department of Pathology, Brigham and Women's Hospital, Harvard Medical School, Boston, MA, USA; \\ ${ }^{d}$ Harvard Medical School, Boston MA 02115, USA \\ Corresponding address: Atul B. Shinagare, MD, Department of Imaging, Dana-Farber Cancer Institute, \\ 450 Brookline Ave, Boston, MA 02115, USA. \\ Email: ashinagare@partners.org
}

Date accepted for publication 23 August 2012

\begin{abstract}
This article reviews the typical and atypical locations, imaging findings, local recurrence, and metastatic pattern of malignant peripheral nerve sheath tumors (MPNSTs). MPNSTs are rare soft tissue sarcomas, commonly occur in extremities, and are often associated with neurofibromatosis. Distinction between benign and malignant tumors can be challenging on imaging. MPNSTs have a poor prognosis; however, rhabdomyoblastic differentiation (malignant triton tumor), which has imaging features similar to MPNSTs, is associated with even more aggressive behavior.
\end{abstract}

Keywords: Malignant peripheral nerve sheath tumor; neurofibromatosis; malignant triton tumor; MRI; PET/CT.

\section{Introduction}

Malignant peripheral nerve sheath tumors (MPNSTs) are malignant neuroectodermal neoplasms that arise from Schwann cells, accounting for $5-10 \%$ of all soft tissue sarcomas $^{[1]}$. Although they may arise sporadically, $20-40 \%$ occur in association with neurofibromatosis type $1(\mathrm{NF} 1)^{[1]}$ (Fig. 1). Sporadic MPNSTs typically occur in the 5 th decade ${ }^{[1]}$, although MPNSTs associated with NF1 occur earlier, at an average age of around 30 years ${ }^{[2]}$. There is no gender predilection ${ }^{[1]}$. Patients commonly present with an enlarging mass with or without pain or other neurologic symptoms ${ }^{[1]}$. Pathologically, MPNSTs are typically composed of spindle cells arranged in a fascicular growth pattern; mitotic rate and extent of tumor necrosis are variable ${ }^{[1]}$. MPNSTs are aggressive, with reported 5-year survival ranging from $34 \%$ to $60 \%{ }^{[1]}$. MPNST with heterologous rhabdomyoblastic (skeletal muscle) differentiation is widely known as malignant triton tumor (MTT). MTTs account for around 5\% of MPNSTs and are associated with poorer outcomes than conventional MPNSTs ${ }^{[2]}$. In this review, we describe the imaging findings, various locations and metastatic pattern of MPNSTs, with an emphasis on differences between MPNST and MTT.

\section{Location}

MPNSTs can develop anywhere in the body, mainly along the major nerve roots ${ }^{[3]}$. Primary tumors most commonly occur in the extremities (45-59\%) (Figs. 1-3), followed by the trunk (17-34\%) (Fig. 4), and head and neck (19-24\%) (Fig. 5) ${ }^{[1,2]}$. Patients with tumors of the extremities have better outcomes compared with other sites of disease, perhaps relating to better local control, whereas tumors of the trunk are associated with poorer outcomes, mainly because of locoregional failures ${ }^{[4]}$. Tumors in the extremities are more likely to present as a painful mass, whereas tumors elsewhere are more likely to be asymptomatic ${ }^{[5]}$.

\section{Imaging features}

On imaging, MPNSTs usually present as a non-specific soft tissue mass (Figs. 2, 3). Their ovoid appearance and location along a nerve should prompt inclusion of a neurogenic tumor in the differential diagnosis ${ }^{[3]}$ (Fig. 3). On ultrasonography, they appear as large, usually elongated, hypoechoic masses (Fig. 6). On computed tomography (CT), these tumors have low attenuation, possibly due to fat entrapment, high lipid content of Schwann cell

This paper is available online at http://www.cancerimaging.org. In the event of a change in the URL address, please use the DOI provided to locate the paper. 


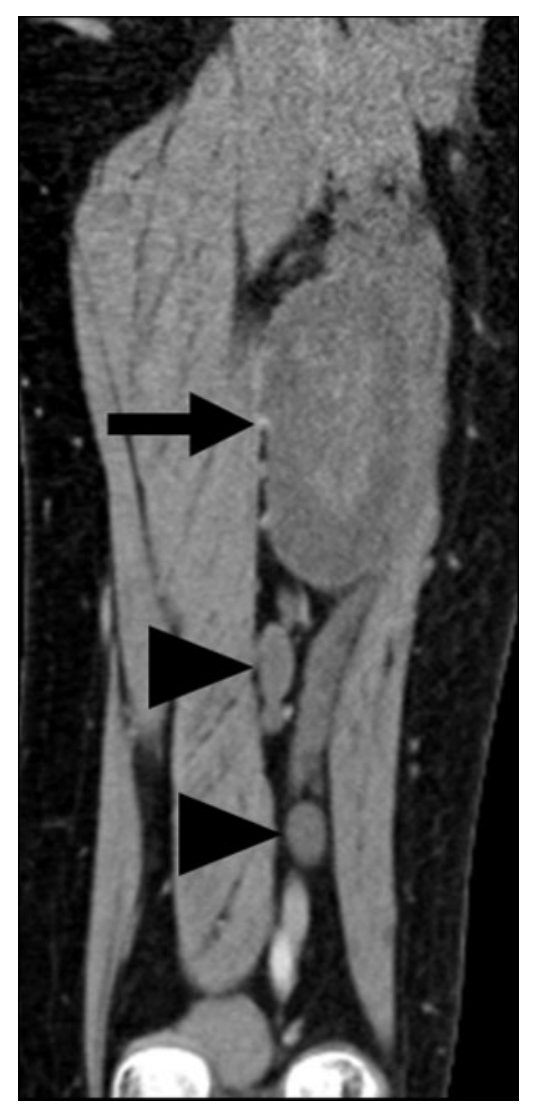

Figure 1 A 38-year-old woman with NF-1 who presented with pain in the left thigh. Coronal contrast-enhanced CT of the left thigh shows an intermuscular well-defined low attenuation mass along the course of the femoral nerve (arrow), with a central area of relatively high attenuation. Histopathology revealed MPNST. Multiple smaller neurofibromas are also seen (arrowheads). myelin, and cystic areas due to hemorrhage or necro$\operatorname{sis}^{[5,6]}$ (Fig. 5a). On magnetic resonance imaging (MRI), MPNSTs are usually isointense to muscle on T1-weighted images and typically hyperintense on T2-weighted images with fascicular appearance ${ }^{[6]}$ (Figs. 3, 7). On positron emission tomography (PET)/ CT, MPNSTs present as fluorodeoxyglucose (FDG)-avid masses (Fig. 3). Although benign nerve sheath tumors can also have mildly increased FDG uptake, uptake in MPNSTs is higher than benign neurogenic tumors (mean maximum standardized uptake value $\left(\mathrm{SUV}_{\max }\right) 8.5$ versus 1.5$)^{[7]}$ (Fig. 3e).

\section{Signs of malignancy in peripheral nerve sheath tumors}

Imaging features of MPNSTs are non-specific and pathologic correlation is always needed for diagnosis. MPNSTs should be considered in the differential of an elongated mass along major nerves. The greatest challenge on imaging is differentiation between benign and malignant nerve sheath tumors.

On plain radiographs, involvement of adjacent osseous structures may suggest malignancy, however sensitivity of radiography is very limited ${ }^{[6]}$. On CT, high attenuation and heterogeneous appearance secondary to necrosis and hemorrhage is commonly seen in malignant tumors $^{[5,6]}$ (Figs. 1, 5). MRI is superior to CT when comparing benign versus malignant peripheral nerve sheath tumors, especially in NF1 patients ${ }^{[6]}$. On MRI, features suggestive of malignancy include rapidly increasing size, heterogeneity on T1-weighted images (Fig. 4), and the presence of intratumoral hemorrhage or necrosis (Figs. 2, 5) with peripheral enhancement (Fig. 4) ${ }^{[3,8-10]}$. (a)

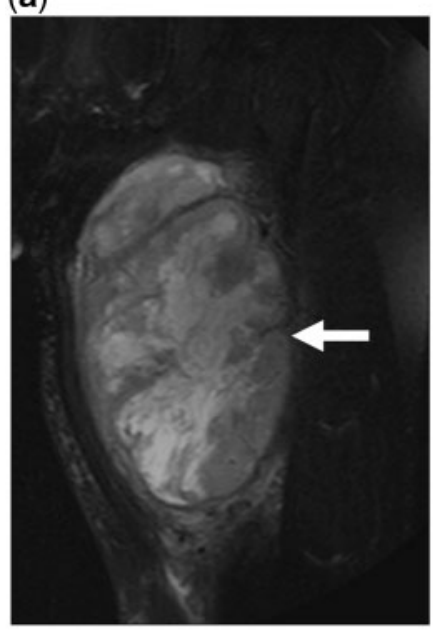

(b)

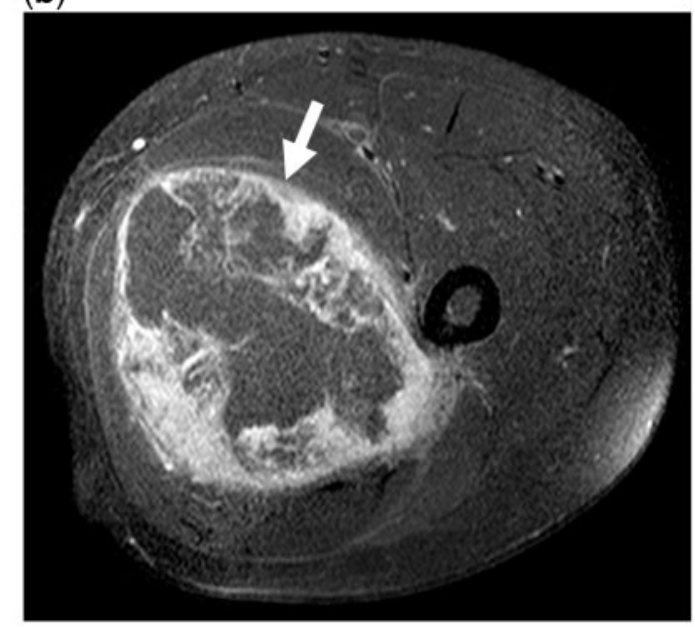

Figure 2 A 35-year-old man presented with swelling of the left thigh. (a) Coronal T2-weighted MR image shows that the mass (arrow) is predominantly hyperintense, with a central markedly hyperintense area, which likely represents necrosis. (b) Axial contrast-enhanced T1-weighted image shows a large central area of necrosis with peripheral rim of enhancement (arrow). 
(a)

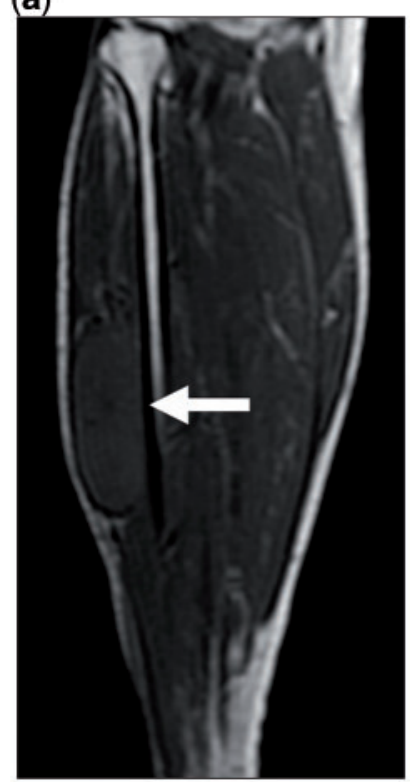

(b)

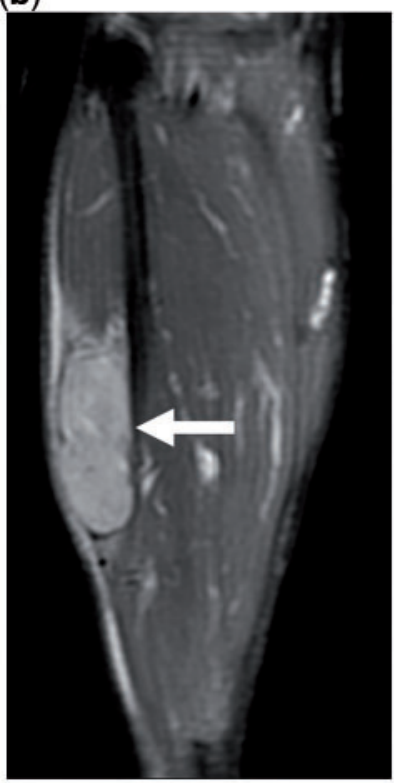

(c)

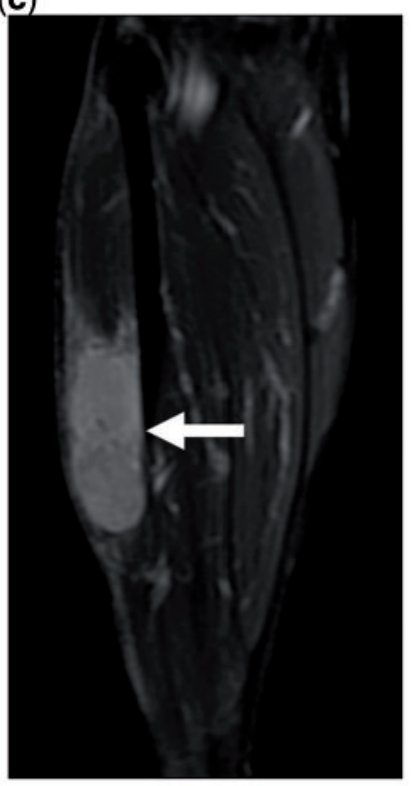

(d)

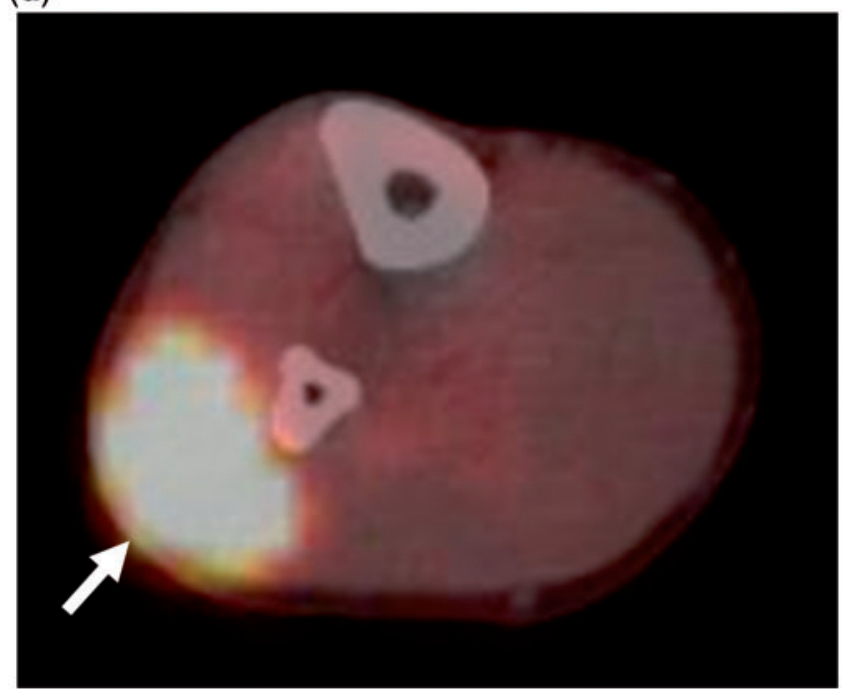

Figure 3 A 62-year-old man with NF-1 presented with increasing size of right calf mass. (a) Coronal T1-weighted MR image shows a well-defined mass (arrow) that is isointense to adjacent muscle. (b) On a coronal T2-weighted MR image, the mass (arrow) is hyperintense compared with the muscles. (c) Coronal contrast-enhanced T1-weighted image shows moderate homogeneous enhancement (arrow). Note that there is no involvement of the underlying bone. (d) Axial fused FDG-PET/CT images show an intensely FDG-avid mass (arrow, $S V_{\max }$ 10.8). (e) Coronal maximum intensity projection (MIP) image shows the FDG-avid mass in the calf (arrow) and several smaller, scattered, less avid lesions (arrowheads) that represent neurofibromas. Notice the difference in the FDG avidity between MPNST and neurofibromas.

Intralesional hemorrhage and necrosis often result in inhomogeneous enhancement (Figs. 2, 4, 5) ${ }^{[10]}$. Infiltrative margins and surrounding edematous changes may be seen ${ }^{[3,8]}$.

Li et al. ${ }^{[3]}$ suggested that larger size and infiltrative margin on MRI indicates malignancy, whereas split fat sign (intact fat planes around the mass) is more indicative of benign tumors, although Wasa et al. ${ }^{[8]}$ found that
MPNSTs often have well-defined margins (Figs. 1, 4, 7). Previously, a target sign, a central hypointense region seen on T2-weighted images, was implicated in malignancy ${ }^{[11]}$ (Fig. 7), but again, recent studies have not corroborated this ${ }^{[8,10]}$. Based on increased FDG uptake associated with malignant transformation, PET/CT reportedly has $72 \%$ specificity for diagnosis of malignancy ${ }^{[7]}$ (Fig. 3e). $\left[{ }^{11} \mathrm{C}\right]$ Methionine-PET may further 


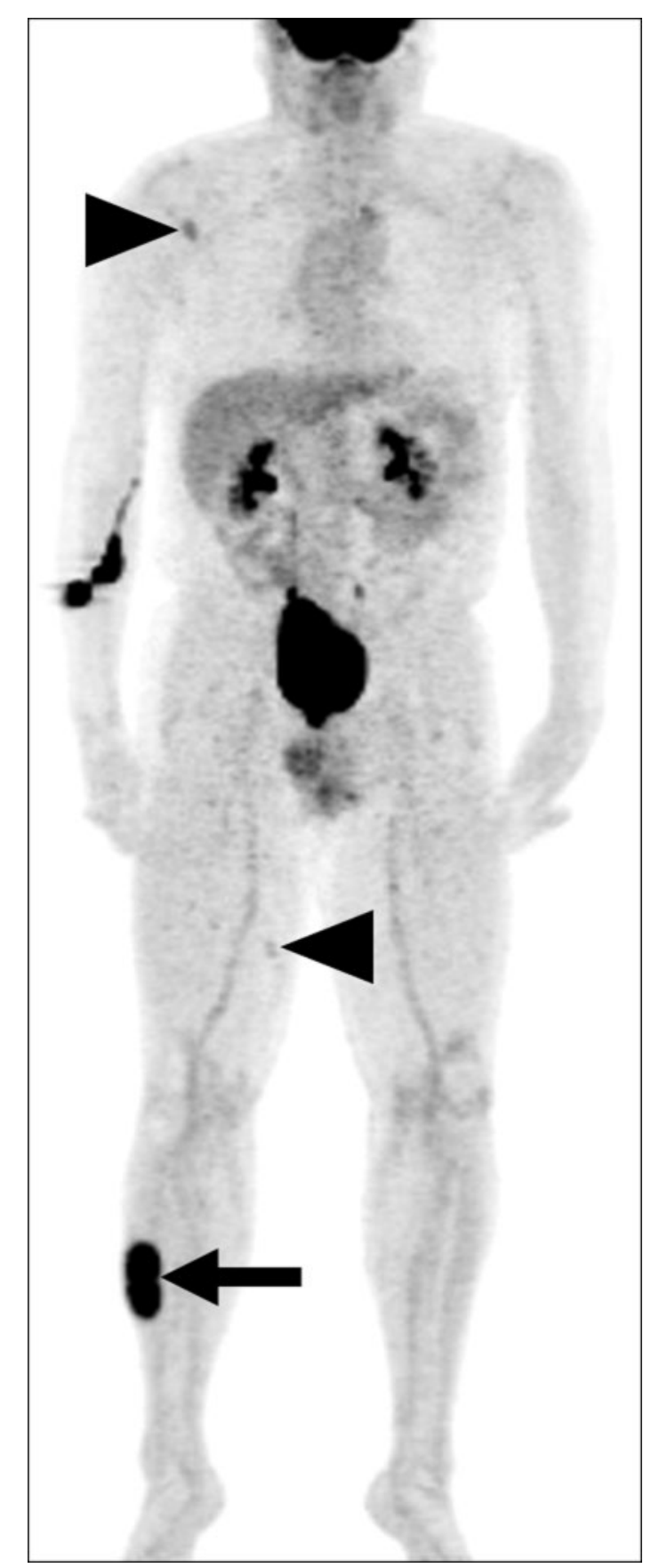

Figure 3 Continued.

increase the specificity to $91 \%$. Given this moderate to high specificity in the detection of MPNSTs, use of PET/ $\mathrm{CT}$ has been recommended for biopsy planning ${ }^{[7]}$.

\section{Management}

Treatment of MPNSTs relies on radical surgical resection to remove the tumor in its entirety and achieve negative margins. Radiation therapy is sometimes given as an adjunct to surgery to improve local control, with Anghileri et al. ${ }^{[4]}$ showing that lack of radiation therapy

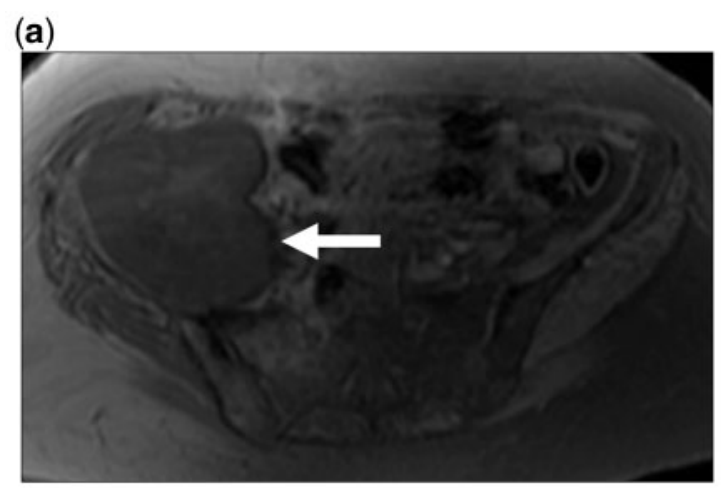

(b)

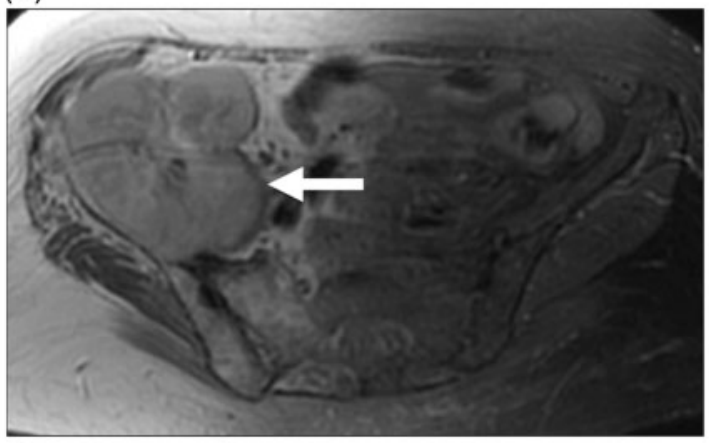

(c)

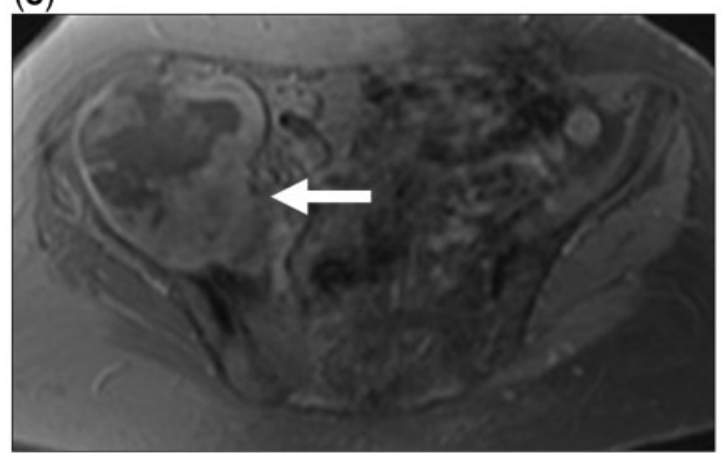

Figure 4 A 75-year-old woman with an incidentally detected mass in the right iliac fossa, proved on histopathology to be an MPNST. (a) An axial T1-weighted MR image shows a heterogeneous isointense mass in the right iliac fossa (arrow) with a central area of central hyperintensity. (b) The mass is hyperintense to muscle on the T2-weighted image. (c) Contrast-enhanced axial T1-weighted image shows a central non-enhancing component likely representing central necrosis and a peripheral enhancing soft tissue rim (arrow).

results in poorer disease-specific survival. Chemotherapy can be used in selected cases (such as tumor rupture, positive margins, metastases) but improvement in survival has not been demonstrated ${ }^{[1]}$. Multimodality treatment is recommended when treating MPNSTs. Radiation therapy is typically considered for tumors with aggressive features (such as size $\geq 5 \mathrm{~cm}$, high grade, and positive margins after surgery) and adjuvant chemotherapy may 
(a)

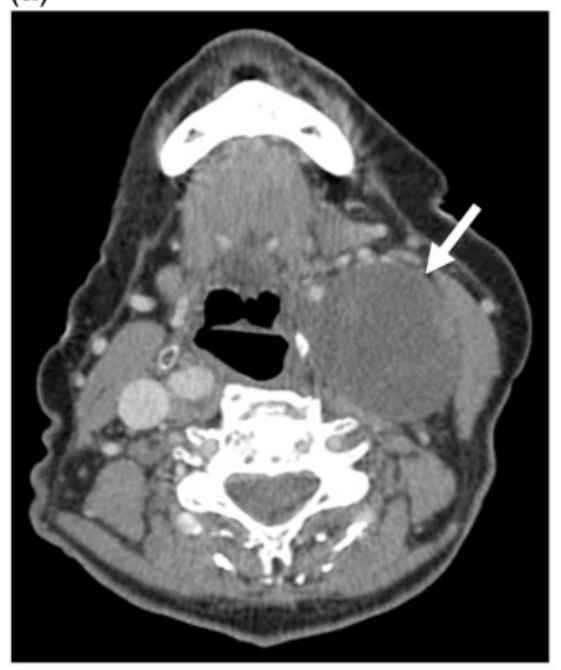

(b)

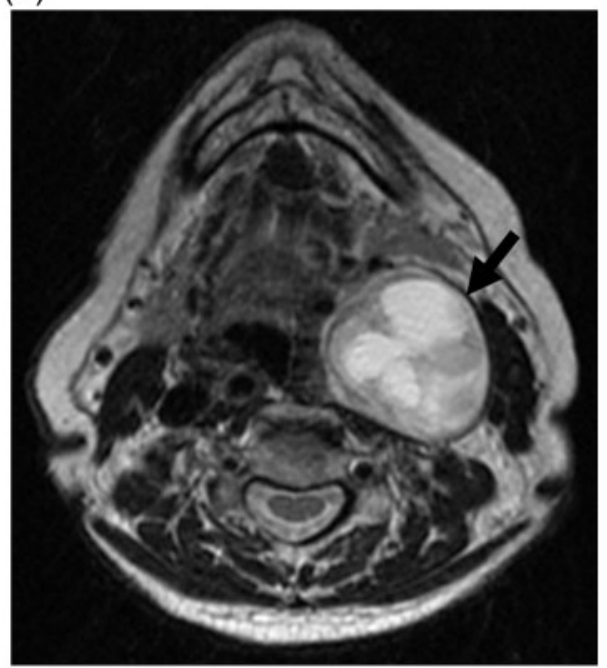

(c)

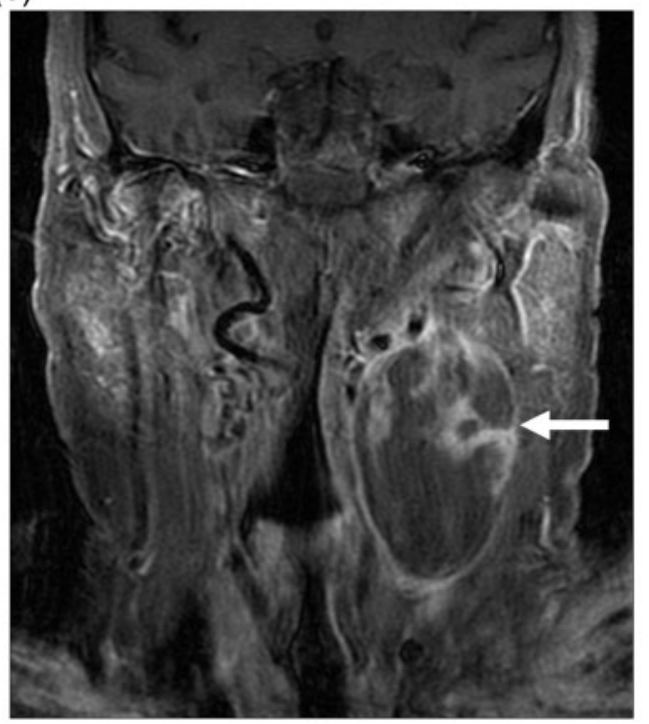

Figure 5 A 76-year-old woman with a history of a left neck mass that had been stable for 19 years, but had started growing 6 months previously. (a) Contrast-enhanced CT image in the axial plane shows a low attenuation mass (arrow) in the left parapharyngeal space, with preserved fat planes anteriorly, laterally and posteriorly. (b) Axial T2-weighted MR image again demonstrates a well-defined mass (arrow) with central T2-hyperintense necrotic areas. (c) Coronal contrastenhanced T1-weighted image shows predominant central necrosis and a thin peripheral rim of enhancement (arrow).

also be considered, particularly for younger patients with these features ${ }^{[1]}$. PET/CT may be used for post-treatment follow-up (Fig. 8).

\section{Malignant triton tumors}

MTTs are defined as MPNSTs with heterologous rhabdomyoblastic differentiation ${ }^{[12]}$. MTTs were first described in 1938 but not so-named until 1973; the name is based on the triton salamander, due to its ability to regenerate limbs and its ability to grow both neural and muscle components from a transplanted sciatic nerve ${ }^{[13]}$. Pathologically, MTTs show the same typical features as high-grade MPNSTs but are distinguished by the presence of cells with skeletal muscle differentiation within the tumor, which usually account for only a minor component ${ }^{[12]}$. Immunohistochemical staining for skeletal muscle markers such as desmin and myogenin can be used to support the diagnosis ${ }^{[12]}$.

The literature on imaging features of MTT is scarce, mainly in the form of isolated case reports ${ }^{[14-16]}$. MTTs typically present as large masses (Fig. 9). Based on the clinical literature, the average age at diagnosis for MTTs is 30 years ${ }^{[12]}$. MTTs are typically larger than MPNSTs, with an average size of $9.0 \mathrm{~cm}$ compared with $6.0 \mathrm{~cm}$ for MPNSTs ${ }^{[1,12]}$. At our institution, we have seen over 


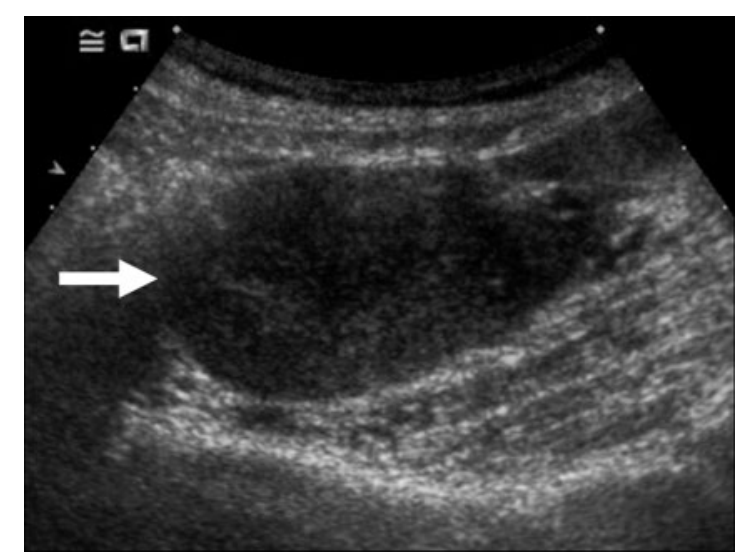

Figure 6 A 72-year-old man with incidentally detected MPNST along the left psoas muscle. Ultrasound image shows a well-delineated, elongated, hypoechoic mass (arrow) without any distinctive features.

20 cases of MTT over the past decade, and in our experience, MTTs cannot be reliably differentiated from other MPNSTs.

\section{Local recurrence and metastasis}

The local recurrence rate of MPNSTs varies from $22 \%$ to $42 \%^{[1]}$; metastases reportedly occur in $20-30 \%$ of patients ${ }^{[1,4]}$. Predictors of local recurrence include positive margin status, trunk tumor site, and large tumor size $^{[4]}$. Metastatic spread is most common to the lungs ${ }^{[1]}$ (Fig. 10). Common extrapulmonary metastases include, but are not limited to, bone (Fig. 10), liver (Fig. 10), peritoneum (Fig. 10), and the central nervous system ${ }^{[1,4]}$. Lymph node metastasis is rarely seen (Fig. 10). PET/CT is useful to detect distant metasta$\operatorname{ses}^{[7]}$ (Fig. 10). On CT or MRI, the radiographic characteristics of metastases often mimic those of the primary lesion.

Predictors of distant metastases include large tumor size, high tumor grade and local recurrence ${ }^{[1,4]}$. Tumor size $\geq 5 \mathrm{~cm}$, local recurrence, and truncal location are associated with a significantly adverse impact on disease-specific survival ${ }^{[1,4]}$. MPNSTs and MTTs in patients with NF1 have a poorer prognosis than sporadic tumors, with a reported 5-year survival of $15-38 \%$, however reports differ regarding the impact of NF1 on survival $^{[2,4,12]}$. MTTs have a worse prognosis than conventional MPNSTs, with a 5 -year survival rate of $11-15 \%$ versus $34-60 \%^{[1,4,12]}$. A thoracoabdominal location of MTT is associated with increased incidence of local recurrence $^{[12]}$. NF1 status and tumor size $\geq 10 \mathrm{~cm}$ are associated with increased incidence of metastatic disease $^{[12]}$.

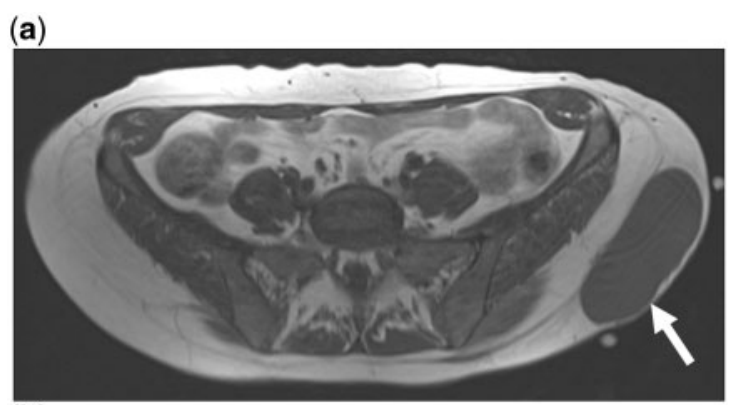

(b)

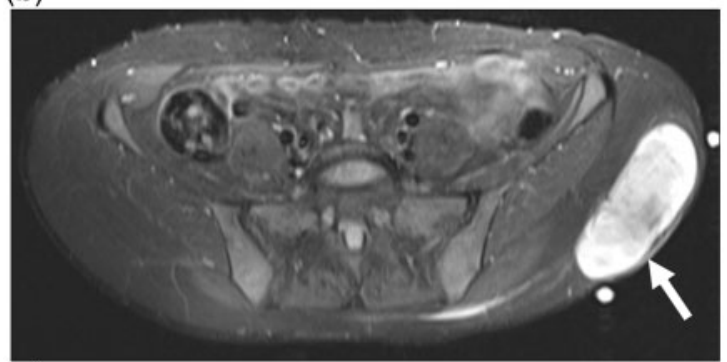

(c)

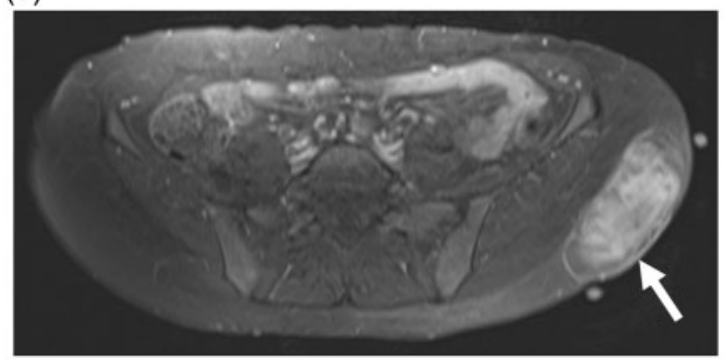

Figure 7 A 47-year-old woman with a history of a mass in the left gluteal region for more than 10 years presented with recent discomfort in the region of the mass; biopsy revealed MPNST. (a) Axial T1-weighted MR image shows a well-defined superficial mass in the left gluteal region (arrow) that is isointense to muscle. (b) On the T2-weighted image, the mass is hyperintense (arrow), typical of neurogenic tumors. (c) Axial contrast-enhanced T1-weighted image shows heterogeneous enhancement.

\section{Conclusion}

MPNSTs often occur in patients with NF1, although they can also arise sporadically, and are associated with a poor prognosis and have a tendency for local recurrence and distant metastasis. These tumors arise anywhere along a peripheral nerve, most commonly in the extremities. Distinction between benign and malignant tumors, which is especially important in patients with NF1, can be challenging on imaging; MRI is the most helpful for this purpose. MPNSTs with heterologous rhabdomyoblastic transformation (MTT) behave more aggressively, with early metastases and poorer prognosis, yet display and imaging pattern similar to MPNSTs. 
(a)

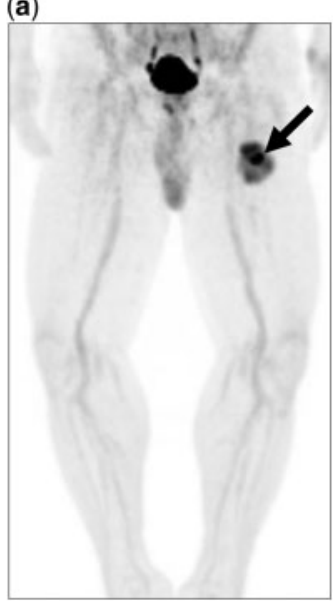

(b)

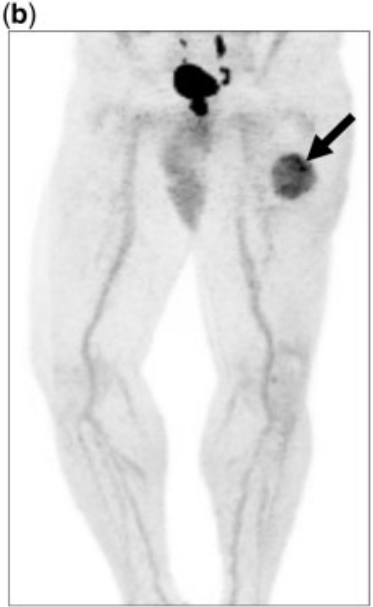

Figure 8 A 56-year-old man with MPNST in the left thigh. (a) Coronal MIP image from pre-treatment PET/CT shows an FDG-avid left thigh mass with $\mathrm{SUV}_{\max }$ of 9.5 (arrow). The patient underwent neoadjuvant radiation. (b) Postradiation PET/CT shows a slight decrease in FDG uptake (SUV $\max 7.1)$, especially in the focus of intense uptake in the superior portion of the mass (arrow).
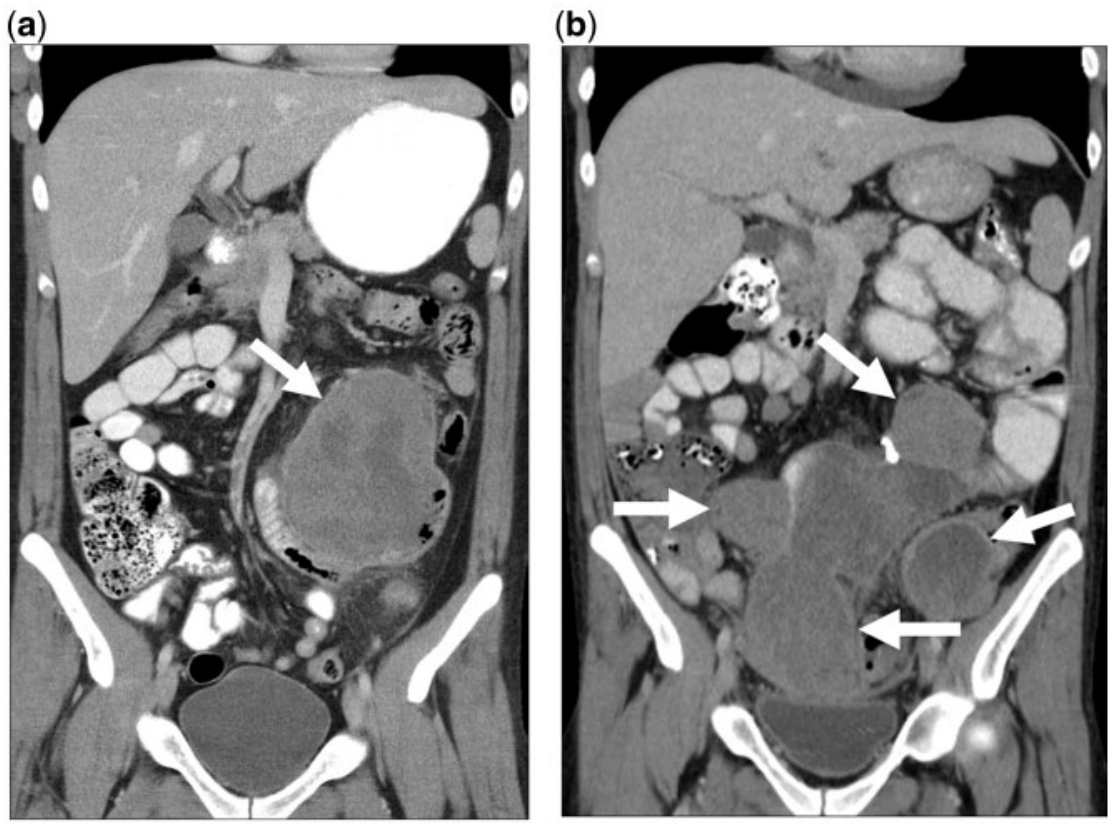

Figure 9 A 31-year-old man with MPNST with rhabdomyoblastic differentiation (MTT). (a) Coronal contrast-enhanced CT shows a large heterogeneous mesenteric mass (arrow) which was resected. (b) Coronal contrast-enhanced CT after 2 months showed extensive disease in the abdomen with multiple large masses (arrows). 
(a)

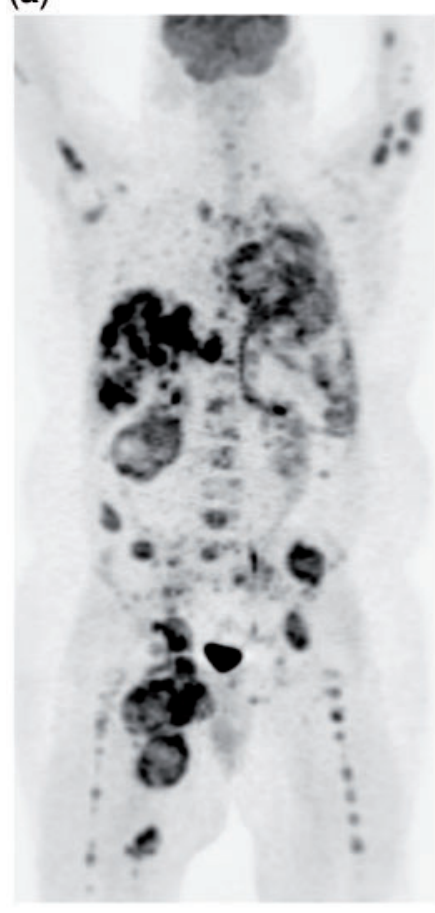

(b)

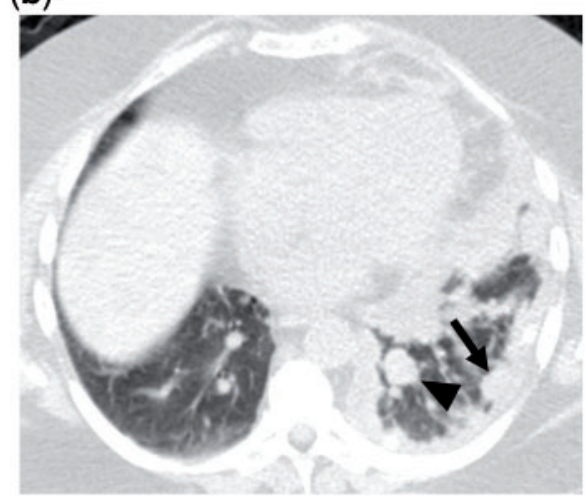

(d)

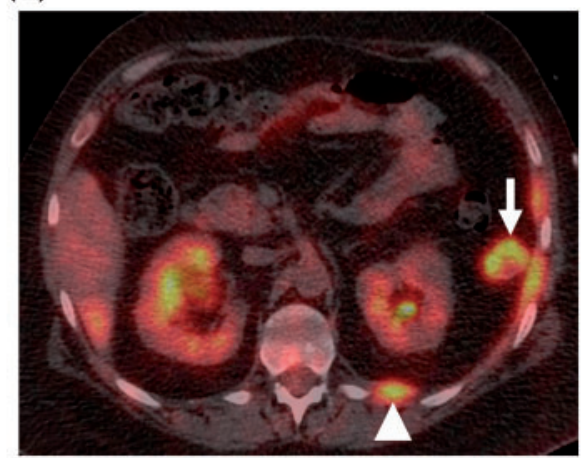

(c)

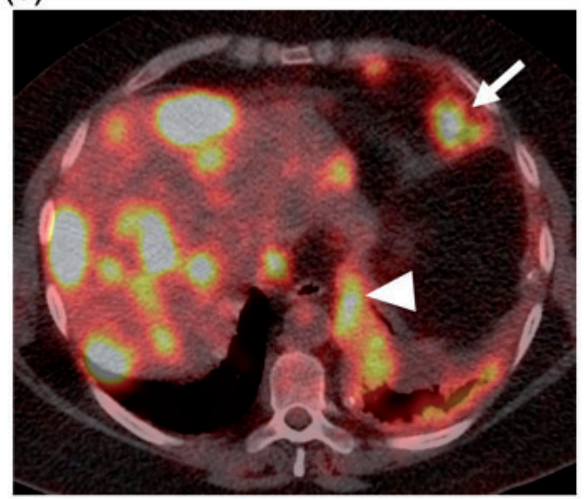

(e)

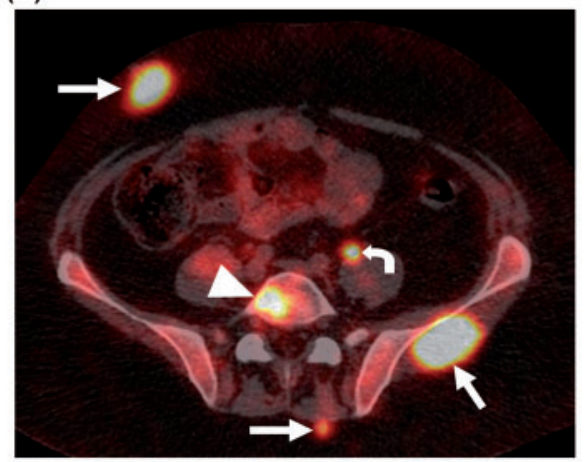

Figure 10 A 34-year-old man with metastatic MTT, originally in the right thigh. (a) MIP image from FDG-PET/CT shows extensive metastatic disease involving the lungs, pleura, lymph nodes, liver, peritoneum, bones and soft tissue. (b) Axial CT image in lung windows from the same PET/CT shows a pulmonary nodule (arrowhead) and extensive pleural thickening (arrow). (c) Fused axial PET/CT image shows multiple intensely FDG-avid liver metastases, epiphrenic lymph node (arrow) and pleural nodularity (arrowhead). (d) Fused axial PET/CT image again shows a pleural nodule (arrowhead) and a peritoneal metastasis (arrow). (e) Fused axial PET/CT image demonstrates soft tissue metastases (arrows) and osseous metastasis (arrowhead). Physiologic uptake is noted within the left ureter (curved arrow).

\section{References}

[1] Stucky CC, Johnson KN, Gray RJ, et al. Malignant peripheral nerve sheath tumors (MPNST): the Mayo Clinic experience. Ann Surg Oncol 2012; 19: 878-885. doi:10.1245/s10434-011-1978-7. PMid:21861229.

[2] Ramanathan RC, Thomas JM. Malignant peripheral nerve sheath tumors associated with von Recklinghausen's neurofibromatosis. Eur J Surg Oncol 1999; 25: 190-193. doi:10.1053/ ejso.1998.0625. PMid:10218464.

[3] Li CS, Huang GS, Wu HD, et al. Differentiation of soft tissue benign and malignant peripheral nerve sheath tumors with magnetic resonance imaging. Clin Imaging 2008; 32: 121-127. doi:10.1016/j.clinimag.2007.05.006. PMid:18313576.

[4] Anghileri M, Miceli R, Fiore M, et al. Malignant peripheral nerve sheath tumors: prognostic factors and survival in a series of patients treated at a single institution. Cancer 2006; 107: 1065-1074. doi:10.1002/cncr.22098. PMid:16881077.

[5] Levy AD, Patel N, Dow N, Abbott RM, Miettinen M, Sobin LH. From the archives of the AFIP: abdominal neoplasms in patients with neurofibromatosis 1: radiologic-pathologic correlation. Radiographics 2005; 25: 455-480. doi:10.1148/rg.252045176. PMid:15798063.

[6] Murphey MD, Smith WS, Smith SE, Kransdorf MJ, Temple HT. From the archives of the AFIP. Imaging of musculoskeletal neurogenic tumors: radiologic-pathologic correlation. Radiographics 1999; 19: 1253-1280. PMid:10489179.

[7] Bredella MA, Torriani M, Hornicek F, et al. Value of PET in the assessment of patients with neurofibromatosis type I. AJR 2007; 189: 928-935. doi:10.2214/AJR.07.2060. PMid:17885067.

[8] Wasa J, Nishida Y, Tsukushi S, et al. MRI features in the differentiation of malignant peripheral nerve sheath tumors and neurofibromas. AJR 2010; 194: 1568-1574. doi:10.2214/ AJR.09.2724. PMid:20489098.

[9] Mautner VF, Friedrich RE, von Deimling A, et al. Malignant peripheral nerve sheath tumors in neurofibromatosis type 1 : MRI supports the diagnosis of malignant plexiform neurofibroma. Neuroradiology 2003; 45: 615-625.

[10] Van Herendael BH, Heyman SRG, Vanhoenacker FM, et al. The value of magnetic resonance imaging in the differentiation between malignant peripheral nerve-sheath tumors and non-neurogenic malignant soft-tissue tumors. Skeletal Radiol 2006; 35: 745-753. doi:10.1007/s00256-006-0160-y. PMid:16775712.

[11] Bhargava R, Parham DM, Lasater OE, Chari RS, Chen G, Fletcher BD. MR imaging differentiation of benign and malignant peripheral nerve sheath tumors: use of the target sign. Pediatr Radiol 1997; 27: 124-129. doi:10.1007/s002470050082. PMid:9028843.

[12] McConnell YJ, Giacomantonio CA. Malignant triton tumors-complete surgical resection and adjuvant radiotherapy 
associated with improved survival. J Surg Oncol 2012; 106: 51-56. doi:10.1002/jso.23042. PMid:22253011.

[13] Brooks JS, Freeman M, Enterline HT. Malignant "Triton" tumors. Natural history and immunohistochemistry of nine new cases with literature review. Cancer 1985; 55: 2543-2549. doi:10.1002/1097-0142(19850601)55:11<2543::AIDCNCR2820551105>3.0.CO;2-4. PMid:3922610.

[14] Han DH, Kim DG, Chi JG, Park SH, Jung HW, Kim YG. Malignant triton tumor of the acoustic nerve. Case report. J Neurosurg 1992; 76: 874-877. PMid:1564550.
[15] Victoria L, McCulloch TM, Callaghan EJ, Bauman NM. Malignant triton tumor of the head and neck: a case report and review of the literature. Head Neck 1999; 21: 663-670. doi:10.1002/(SICI)1097-0347(199910)21:7<663::AIDHED12>3.0.CO;2-4. PMid:10487955.

[16] Yimaz MR, Bek S, Bekmezci T, Gokduman C, Solak A AS. Malignant triton tumor of the lumbar spine. Spine 2004; 29: E399-E401. PMid:15371720. 